\title{
Effect of ACE inhibitors on the atrial natriuretic peptides in heart failure
}

Atrial natriuretic peptide (ANP) was sequenced 10 years ago. There was a huge flurry of excitement, interest, and research activity that waned after 5 years. Since then ANP has been found to belong to a family of natriuretic peptides (ANP, brain natriuretic peptide, and C type natriuretic peptide). This has led to a resurgence of interest in their diagnostic and therapeutic potential.

Yet, despite 10 years of research many fundamental questions remain unanswered. One area of uncertainty is what regulates the synthesis and release of ANP. Two papers in this month's British Heart fournal (pages 521 and 528) examine the mechanisms by which ACE inhibitors alter plasma ANP concentrations in chronic heart failure. ${ }^{12}$ Both studies concentrate on the first 24 hours of treatment, which makes them somewhat inconclusive. In both studies short-term treatment with an ACE inhibitor was given and the changes in pulmonary capillary wedge pressure (PCWP) were examined in relation to the coincidental changes in plasma ANP concentrations. This is not an unreasonable approach but it does not take into account the fact that ANP changes may lag behind haemodynamic changes. To avoid this problem, it is better to examine the relation between ANP changes and haemodynamic changes when both are at steady state. Both papers, however, do follow the hormonal changes and the haemodynamic changes for 24 hours after the short-term treatment, which does partially compensate for the lack of proven steady state.

Despite the above caveat both papers produce some thought provoking data. Berglund et al did a comprehensive study of 38 patients with chronic heart failure who were given three different doses of ramipril. Three hours after the ACE dose, both ANP and PCWP fell so that the ANP/PCWP ratio remained constant. However, at 24 hours, the ANP/PCWP ratio increased significantly, principally because ANP plasma concentrations returned to their baseline value while PCWP remained reduced. Berglund et al cannot precisely explain this finding but they not unreasonably suggest that some resetting may have occurred in the relation between atrial pressure and ANP.
Yoshimura et al measured not only ANP but also B type or brain natriuretic peptide (BNP) after administration of alacepril (a captopril pro-drug) in controls and those with congestive heart failure. Unfortunately there was no placebo day with either group of patients so some doubt remains about how much of the change was attributable to the treatment and how much to time. Nevertheless, the data are very clearly presented and show (fig 4) that ANP falls acutely (at 2 hours) in parallel with the PCWP fall whereas BNP falls later (at 8-12 hours) and that the fall in BNP is dissociated from the earlier PCWP fall. This suggests, as is thought, that the secretion of ANP is regulated by atrial pressure whereas BNP is not. Yoshimura et al did not calculate the ANP/PCWP ratio and therefore we cannot tell whether they found the same increase in the ANP/PCWP ratio at 24 hours as Berglund et al did.

It is impossible in studies of patients such as these to be precise about which haemodynamic factors are causing ANP and BNP release because it is difficult to know when both the haemodynamic and the hormonal factors are at steady state. Nevertheless, in these two papers, we have good evidence that atrial pressure determines ANP concentrations and that some resetting of the ANP/atrial pressure relation occurs after acute ACE inhibition. Furthermore BNP seems to be regulated differently and not apparently by atrial pressure because it falls some time after the ANP fall has disappeared.

Department of Clinical Pharmacology, ALLAN D STRUTHERS Ninewells Hospital and Medical School, Dundee DD1 $9 S Y$

1 Berglund H, Nyquist O, Beermann B, Jensen-Urstad $M$, Theodorsson E. Influence of angiotensin converting enzyme inhibition on the atrial natriuretic peptide/atrial pressure relationship in heart failure. $\mathrm{Br}$ Heart 7 1994;72:521-527.

2 Yoshimura $M$, Yasue $H$, Tanaka $H$, Kikuta $K$, Sumida $H$, Kato $H$, et al. Responses of plasma levels of A-type natriuretic peptide and B-type natriuretic peptide to alacepril, an angiotensin-converting enzyme natriuretic peptide to alacepril, an angiotensin-converting enzyme inhibitor in patient 